\title{
On the spider fauna (Arachnida: Aranei) of Udmurt Republic
}

\section{К фауне пауков (Arachnida: Aranei) УАмуртской Респубцики}

\author{
A.N. Sozontov*, S.L. Esyunin ${ }^{* *}$

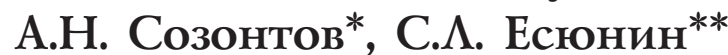 \\ * Department of Ecology of Animals, Udmurt State University, Universitetskaya Street 1/1, Izhevsk 426034 Russia
**Department of Zoology, Perm State University, Bukireva Street 15, Perm 614600 Russia
* Удмуртский государственный университет, ул. Университетская, 1\1, г. Ижевск 426034 Россия
** Кафедра зоологии беспозвоночных животных, Пермский государственный университет, ул. Букирева 15, г. Пермь 614600
Россия.
}

KEY WORDS: spider, fauna, Udmurtia.

КЛЮЧЕВЫЕ СЛОВА: пауки, фауна, Удмуртия.

ABSTRACT. A check-list of 195 spider species of 105 genera and 24 families collected from Udmurtia is provided. Of them 125 species are new to Udmurtia. Local records of all the recorded species are given.

РЕЗЮМЕ. Для Удмуртии приведен список из 195 видов из 105 родов 24 семейств пауков. Из них 125 видов впервые указаны с территории Удмуртии. Даны точки находок пауков.

\section{Introduction}

Udmurtia (=Udmurt Republic) is situated in the east part of the Russian Plain and lies within the boreal and subboreal zones which include the following three subzones: the southern taiga, the coniferous-broadleaved forests dominated by conifers and the broadleaved-coniferous forests dominated by broadleaved trees (Map) [Baranova, 2002].

The spider fauna of Udmurtia remains poorly studied. The first record of a single species, Allohogna singoriensis (Laxman, 1770), was made by L.K. Krulikovskiy [1892, 1908] for Kirov Area. Although three more species were reported by Krulikovskiy [1908] from "Vyatka Government", their exact localities are unknown to us because we failed to obtain the original paper; the information is thus based on Shernin [1971]. Therefore, it is not clear whether these records were made from Udmurtia or from Kirov Area.

The first and the latest major paper concerning the spiders of Udmurtia is that by Zubko \& Roshchinenko [1981], who published a list of 69 species of 16 families. On the basis of these data, three species, Allohogna singoriensis, Arctosa perita (Latreille, 1799) and Eresus kollari Rossi, 1846, were included in the "Red book of Udmurt Republic" [Zubko, 2001]. Thus, by the beginning of our research 70 spider species had been known from Udmurtia. Records of 21 species have not been confirmed by our own data.
In this paper, we have summarized both the literature-derived and original data on spiders of Udmurtia. To date, this spider fauna consists of 195 species of 105 genera and 24 families. Compared to the spider faunas of neighbouring Mari-El Republic, Tartaria, Chuvashia (389, 254 and 238 species, correspondingly [Krasnobaev, 2004]), Kirov (296 species [Esyunin et. al., 2011]) and Perm areas (542 species Esyunin, unpublished data), the species number known from Udmurtia (195 species) is relatively low.

\section{Material and methods}

This study is based on the spider collections made by the authors in several localities of Udmurtia in 2007 2010 , as well as the one by A.V. Il'ina from the vicinity of Cheptsa Village in 2008. Detailed information concerning the studied localities is presented below.

Description of the collecting localities (Map)

South taiga subzone

1. Cheptsa Village, Kez District, VII.2008.

2. Starye Zyatcy Village, Yakshur-Bod'ya District, V.2010.

3. $25 \mathrm{~km}$ from Izhevsk to Yakshur-Bod'ya, Yakshur-Bod'ya District, IX.2009.

4. $16 \mathrm{~km}$ from Izhevsk to Yakshur-Bod'ya, Yakshur-Bod'ya District, X.2009.

5. Volozhka Village, Zav'yalovo District, VI.2010.

6. Izhevsk City, VII \& IX.2009.

Subboreal (=nemoral) zone

7. National Park «Nechkinskiy» (grey color in map), Zav'yalovo, Votkinsk \& Sarapul Districts:

a. Doksha Village, Zav'yalovo District, VI.2008, VI.2009, VI, VIII \& IX.2010; 
b. Siva biostation, near PereFvoznoe Village, Votkinsk District, VI.2007, VI.2008, VIII.2009, VIII.2010;

c. Novyi Village, Votkinsk District, VI.2008, VI \& VIII.2009, VI-VII.2010;

d. Environs of Volkovskii Village, Votkinsk District, VI.2009, VI.2010;

e. Gol'yany Village, Zav'yalovo District, VIII.2010;

f. Makarovo Village, Zav'yalovo District, VI.2009;

g. Nechkino Village, Sarapul District, VI \& VIII.2009.

8. Parshur Village, Mozhga District, VII.2008.

9. Stariy Utchan Village, Alnashy District, VII.2009.

10. Pirogovo Village, Alnashy District, VII.2009.

The studied material is shared between the collections of the Udmurtskiy State University, Izhevsk, Russia and the Department of Zoology of the Perm State University, Perm, Russia (PSU).

Abbreviations used in the text: ANS - A.N Sozontov, AVI - A.V. Il'ina, juv. — juv. specimens.

\section{AGELENIDAE}

Tegenaria domestica (Clerck, 1758)

Udmurtia [Zubko \& Roshchinenko, 1981]: in house.

\section{ANYPHAENIDAE}

Anyphaena accentuata (Walkenaer, 1802)

MATERIAL. 1 subadult $\sigma^{7}, 4$ juv. [7]: Siva, small-leaved forest, 10.VIII.2010, leg. ANS

\section{ARANEIDAE}

Aculepeira ceropegia (Walckenaer, 1802)

MATERIAL. 1 \% (PSU-6068) [9], mixed forest, 10.VII.2009, leg. ANS.

Agalenatea redii (Scopoli, 1763)

MATERIAL. 1 subadult + [7]: Novyi, mixed forest, 19.VIII 2009, leg. ANS

Araneus alsine (Walckenaer, 1802)

MATERIAL. 1 subadult $\sigma^{7}$ [7]: Novyi, bottomland oak forest, 21.VI.2009, leg. ANS.

Araneus angulatus Clerck, 1758

MATERIAL. 1 [ [7]: Novyi, dry meadow, 18.VIII.2009, leg. ANS; 1 [ [8], edge of mixed forest, 21.VII.2008, leg. ANS; 1 . Krasnogorskoe District, Krasnogorskoe Village, in a crone of the birch (Betula pendula) standing on the bank of a pond, VIII.2009, K.V. Vasil'ev.

Araneus diadematus Clerck, 1758

MATERIAL. 1 subadult 9 [3], small-leaved forest, 15.IX.2009,

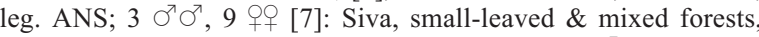
24.VIII.2009, 10.VIII.2010, leg. ANS; 1 subadult $O^{7}$ [7]: Novyi, edge of mixed forest, 21.VI.2009, leg. ANS; 1 juv., same locality, Pinus forest, 17.V.2009, leg. ANS; 19 , same locality, dry meadow on bank of Kama River, 21.VIII.2009, leg. ANS; 4 +o [7]: Gol'yany, bottomland meadow, dry lichen-grass association on sand, 7.VIII.2010, leg. ANS;

Udmurtia [Zubko \& Roshchinenko, 1981]: Picea-Betula \& Alnus forests.

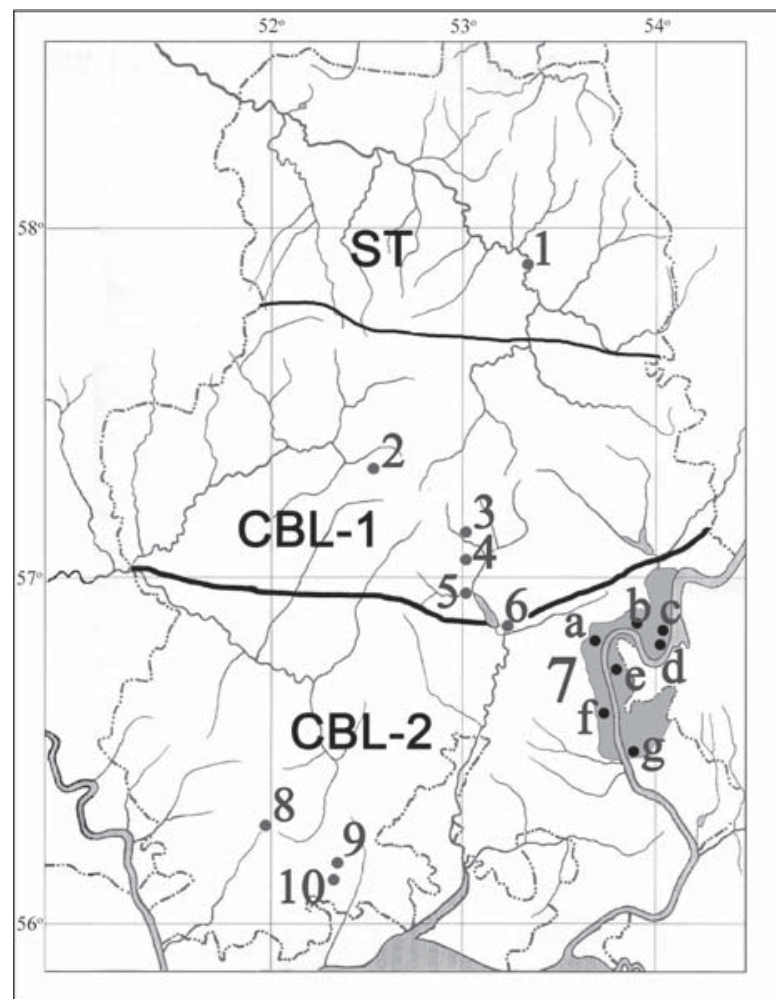

Map. Collecting localities (1-10; see for the explanations given in "Description of the collecting localities") and the scheme of vegetation subzones of the Udmurt Republic. The vegetation zone and subzones: ST - south taiga subzone, CBL - subboreal zone with two subzones: 1 - coniferous-broadleaved forests, 2 broadleaved-coniferous forests.

Карта. Локалитеты (описание смотри в разделе "Description of the collecting localities") и схемы растительных зон и подзон Удмуртской Республики. Растительные зоны и подзоны: ST подзона южной тайги, CBL - подтаёжная зона с двумя подзонами: 1 - хвойно-широколиственных лесов, 2 - широколиственно-хвойных лесов.

Araneus marmoreus Clerck, 1758

MATERIAL. 1 ㅇ, [1], birch forest, VII.2008, leg. AVIl; 1 ㅇ, [2], kitche-garden, VIII.2009, leg. ANS; 1 subadult $O^{7}$, [7]: Siva, bottomland Quercus forest, 10.VIII.2010, leg. ANS; 1 subadult + , [7]: Novyi, Sphagnum bog, 19.VI.2009, leg. ANS; 1 , same locality and biotope, 18.VIII.2009, leg. ANS; $1 \sigma^{7}$, [7]: Nechkino, bottomland meadow, 09.VI.2009, leg. ANS; $10^{7}, 1$, [8], meadow bog, 21.VII.2008, leg. ANS; 1 O', [9], bog, 7.VII.2009, leg. ANS;

Udmurtia [Zubko \& Roshchinenko, 1981]: Picea-Betula \& Pinus forests.

Araneus quadratus Clerck, 1758

MATERIAL. 1 ㅇ [5], multiherbaceous meadow, VI.2010, leg. ANS; 1 ㅇ [2], building, VIII.2009, leg. ANS; 1 ㅇ, 2 subadult 우오 [7]: Volkovskii, bottomland oak forest, coastal vegetation, 20.VI.2009, leg. ANS.

Araneus sturmi (Hahn, 1831)

MATERIAL. 1 [ [7]: Doksha, bank of river, 20.VI.2008, leg. ANS.

Araniella proxima (Kulczyński, 1885)

MATERIAL. $1 \sigma^{7}$ [7]: Novyi, bog, 18.VI.2008, leg. ANS; 1 웅 [7]: Volkovskii, shrub on edge of bog, 18.VI.2009, leg. ANS; $10^{7}$ 
[7]: Nechkino, Salex thicket on bank, 09.VI.2009, leg. ANS; 1 [9], edge of mixed forest, 8.VII.2009, leg. ANS.

\section{Cercidia prominens (Westring, 1851)} ANS.

MATERIAL. $1 \sigma^{7}$ [7]: Nechkino, Pinus forest, VIII.2009, leg.

Cyclosa conica (Pallas, 1772)

MATERIAL. 1 subadult $\sigma^{7}$ [3], Pinus forest, 15.IX.2009, leg ANS; $2 \sigma^{7} \sigma^{7}, 2$ 우 [7]: Novyi, Pinus forest, 16-17.VI.2009, leg. ANS; 1 [ [9], edge of mixed forest, 8.VII.2009, leg. ANS.

Cyclosa îculata (Walckenaer, 1802) ANS

MATERIAL. 1 ㅇ [7]: Novyi, dry meadow, 198.VI.2008, leg.

Gibbaranea omoeda (Thorell, 1870)

Udmurtia [Zubko \& Roshchinenko, 1981]: Picea-Betula \& Pinus forests.

Hypsosinga heri (Hahn, 1831)

MATERIAL. 3 ㅇ, [7]: Siva, bottomland meadow, 15.VI.2008, leg. ANS; 2 क⿱, [7]: Volkovskii, coastal vegetation, 20.VI.2009, leg. ANS.

Hypsosinga pygmaea (Sundevall, 1831)

MATERIAL. 1 \%, [7]: Siva, bottomland Quercus forest, 15.VI.2008, leg. ANS; 2 O+, [7]: Novyi, dry multiherbaceous meadow \& bottomland oak forest, 21.VI.2009, leg. ANS; $2 \sigma^{7} \sigma^{7}, 2$ 우, [7]: Volkovskii, bog, 12.VI.2010, leg. ANS.

Larinioides cornutus (Clerck, 1757)

MATERIAL. 1 [ [5], multiherbaceous meadow, VI.2010, leg ANS; 9 O $^{7}, 4$ ㅇ [7]: Siva, bottomland Quercus forest \& bottomland meadow, 15.VI.2008, leg. ANS; ; 1 [7]: Novyi, edge of mixed forest, 18.VI.2009, leg. ANS; 2 우, same locality, dry multiherbaceous meadow \& bottomland meadow, 21.VI.2009, leg. ANS 5 우, 1 subadult $\Im^{\top}$ [7]: Volkovskii, bottomland oak forest, bog, coastal vegetation, 18-20.VI.2009, leg. ANS; 1 q [7]: Nechkino, bottomland meadow, 9.VI.2009, leg. ANS

\section{Larinioides ixobolus (Thorell, 1873)}

MATERIAL. 1 [ [7]: Doksha, bank of river, 10.VIII.2010, leg. ANS; 1 [ [2], kitchen-garden, VIII.2009, leg. ANS; 1 [7]: Siva bottomland meadow, VI.2007, leg. ANS; 1 \%, 3 우, same locality, bottomland Quercus forest, wall of shed, 10.VIII.2010, leg. ANS.

Udmurtia [Zubko \& Roshchinenko, 1981]: Picea-Betula \& Pinus forests.

Larinioides patagiatus (Clerck, 1757)

MATERIAL. 1 O [1], kitchen garden, VII.2008, leg. AVIl; 19 [7]: Doksha, bank of river, 23.VI.2009, leg. ANS; 1 万', same locality and biotope, 10.VIII.2010, leg. ANS; 5 우 [7]: Siva, smallleaved forest, 10.VIII.2010, leg. ANS; 1 \%', 3 of [7]: Novyi, earthen bank of a water basin, 19.VIII.2009, leg. ANS; 1 q [7]: Volkovskii, shrub on edge of bog, 18.VI.2009, leg. ANS; 2 우 [7] Nechkino, bottomland meadow, 9.VI.2009, leg. ANS; 1 ㅇ [9], bank of lake, 8.VII.2009, leg. ANS.

Udmurtia [Zubko \& Roshchinenko, 1981]: Picea-Betula \& Pinus forests, forest meadow.

Larinioides suspicax (O.Pickard-Cambridge, 1876) MATERIAL. 2 우 [9], bank of lake, 8.VII.2009, leg. ANS.

Mangora acalypha (Walckenaer, 1802)

MATERIAL. 8 juv. [1], dry multiherbaceous and agricultural meadows, VII.2008, leg. AVIl; 2 우 [7]: Novyi, bog, 19.VI.2009, leg. ANS; 1 \%, 2 , ceous Pinus forest, 10-12.VI.2010, leg. ANS; 2 우, 2 subadult $\sigma^{7} \sigma^{7}, 4$ juv. [7]: Volkovskii, dry meadow, shrub on edge of bog, 18.VI.2009, leg. ANS; 3 우, same locality, dry meadow, 12.VI.2010, leg. ANS

Udmurtia [Zubko \& Roshchinenko, 1981]: Pinus forest, forest meadow.

Singa hamata (Clerck, 1758)

MATERIAL. $1 \sigma^{7}$ [7]: Doksha, bank of river, 11.IX.2010, leg. ANS; $1 \bigcirc^{7}, 1$ [ [7]: Novyi, bottomland meadow \& dry meadow on bank of Kama River, 21.VI.2009, leg. ANS; 1 juv., same locality, Pinus forest, 17.VI.2009, leg. ANS; 1 ऽ, 2 우, same locality, Pinus forest with green moss \& earthen bank of a water basin, 10 16.VI.2010, leg. ANS; 1 juv. [7]: Volkovskii, dry meadow, 12.VI.2010, leg. ANS; 1 ○ [7]: Makarovo, bank of river, 10.VI.2009, leg. ANS; 1 ○', 1 우 [7]: Nechkino, mixed forest, bottomland meadow, 09.VI.2009, leg. ANS; 1 \% [7]: Siva, smallleaved forest, 24.VIII.2009, leg. ANS; 2 우 [10], multiherbaceous meadow, 10.VII.2009, leg. ANS.

\section{Singa nitidula C.L. Koch, 1844}

MATERIAL. 1 [7]: Doksha, bank of river, 23.VI.2009, leg. ANS; 1 + [7]: Siva, bottomland meadow, 10.VIII.2010, leg. ANS; 1 [ [7]: Novyi, Pinus forest, 17.VI.2009, leg. ANS; 1 i same locality and biotope, 11.IX.2010, leg. ANS; $1 \sigma^{7}$ [7]: Makarovo, bank of river, 10.VI.2009, leg. ANS.

Stroemiellus stroemi (Thorell 1875)

MATERIAL. 7 Oొ $^{7}, 2$ 우 (PSU-5315) [1], kitchen garden, VII.2008, leg. AVIl; 1 + [7]: Novyi, mixed forest, litter, 19.VIII.2009, leg. ANS.

\section{CHEIRACANTHIIDAE}

Cheiracanthium erraticum (Walckenaer, 1802)

MATERIAL. 1 (PSU-5309) [1], dry multiherbaceous meadows, VII.2008, leg. AVIl; 4 오 [7]: Novyi, edge of mixed forest, kitchen garden, 16-21.VI.2009, leg. ANS; $1 \sigma^{7}, 1$ ㅇ [7]: Volkovskii, bottomland oak forest, shrub on edge of bog, 18-20.VI.2009, leg. ANS; 1 ㅇ [7]: Gol'yany, bottomland meadow, 7.VIII.2010, leg. ANS.

Cheiracanthium pennyi O. Pickard-Cambridge, 1873

MATERIAL. 1 \& [1], agricultural meadows, VII.2008, leg. AVIl.

\section{CLUBIONIDAE}

Clubiona diversa O. Pickard-Cambridge, 1862

Udmurtia [Zubko \& Roshchinenko, 1981]: forest meadow.

Clubiona lutescens Westring, 1851

MATERIAL. 3 ㅇ [7]: Novyi, mixed forest, litter, 16.VI \& 21.VIII.2009, leg. ANS

Udmurtia [Zubko \& Roshchinenko, 1981]: forest meadow.

Clubiona neglecta O. Pickard-Cambridge, 1862 ANS.

MATERIAL. $1 \sigma^{7}$ [5], multiherbaceous meadow, VI.2010, leg.

Udmurtia [Zubko \& Roshchinenko, 1981]: forest meadow, bank of lake.

Clubiona pseudoneglecta Wunderlich, 1994

MATERIAL. 1 9 [7]: Novyi, dry meadow, 21.VIII.2009, leg. ANS; 1 , same locality, earthen breakage of a water basin, 10.VI.2010, leg. ANS. 
Clubiona reclusa O. Pickard-Cambridge, 1863

MATERIAL. $1 \sigma^{7}$ [7]: Siva, bottomland meadow, 26.VI.2008, leg. ANS; 3 우 [7]: Novyi, dry meadow \& bottomland meadow, 21.VI.2009, leg. ANS; $1 \sigma^{7}$ [7]: Makarovo, bank of river, 10.VI 2009, leg. ANS.

Clubiona similes L. Koch, 1867

Udmurtia [Zubko \& Roshchinenko, 1981]: forest meadow.

Clubiona stagnatilis Kulczynski, 1897

MATERIAL. $10^{7}$ [7]: Novyi, bottomland meadow, 21.VI.2009, leg. ANS; 1 $[9]$, bank of lake, 8.VII.2009, leg. ANS.

Clubiona subsultans Thorell, 1875

MATERIAL. $1 \sigma^{7}, 1$ + [4], mixed forest, 6.X.2009, leg. ANS; 1 ㅇ [7]: Siva, bottomland meadow, 26.VI.2008, leg. ANS.

\section{CORINNIDAE}

Phrurolithus festivus (C.L. Koch, 1835)

MATERIAL. 1 [ [7]: Novyi, Pinus forest, 17.VI.2009, leg. ANS.

\section{CYBAEIDAE}

Argyroneta aquatica (Clerck, 1758)

MATERIAL. 1 \% [7]: Novyi, former river-bed, in water, VI.2009, leg. ANS

Udmurtia [Zubko \& Roshchinenko, 1981]: water ecosystem.

\section{DICTYNIDAE}

Dictyna arundinacea (Linnaeus, 1758)

MATERIAL. $1 \sigma^{7}$ [1], VII.2008, dry multiherbaceous meadow, leg. AVIl; 2 우 [5], multiherbaceous meadow, VI.2010, leg. ANS; 1 ㅇ [7]: Novyi, bottomland Quercus forest, 12.VI.2009, leg. ANS; 1 , same locality, dry meadow, 10.VI.2010, leg. ANS; 3 우 [7]: Volkovskii, bottomland oak forest, 20.VI.2009, leg. ANS; 4 OP, same locality, dry meadow, 18.VI.2009 \& 12.VI.2010, leg. ANS; 1 ㅇ, same locality, bog, 10.VI.2010, leg. ANS; 1 ㅇ [7] Nechkino, mixed forest, 9.VI.2009, leg. ANS.

Udmurtia [Zubko \& Roshchinenko, 1981]: Pinus, Picea-Betula \& Alnus forests.

Dictyna pusilla Thorell, 1856 ANS.

MATERIAL. 1 ㅇ [7]: Siva, bottomland meadow, VI.2010, leg.

Dictyna uncinata Thorell, 1856

MATERIAL. 1 \& [7]: Novyi, earthen breakage of a water basin, 2.VII.2010, leg. ANS

\section{ERESIDAE}

Eresus kollari Rossi, 1846

REMARKS. This species absent in our collection. It is recorded on the basis of the message of D.A. Adakhoskii, who observed three male on bank Kama River coast opposite to Gol'yany Village (Zav'yalovo District) at 02.VI.2006.

Karakulino District [Zubko, 2001: as Eresus cinnaberinus (Oliver, 1789)].

\section{GNAPHOSIDAE}

Berlandina cinerea (Menge, 1872) ANS.

MATERIAL. 1 juv. [7]: Novyi, Pinus forest, 17.VI.2009, leg.

Udmurtia [Zubko \& Roshchinenko, 1981]: fallow land.
Drassodes lapidosus (Walckenaer, 1802)

Udmurtia [Zubko \& Roshchinenko, 1981]: fallow land.

Drassodes villosus (Thorell, 1856)

Udmurtia [Zubko \& Roshchinenko, 1981]: fallow land.

Drassyllus pusillus (C.L. Koch, 1833)

MATERIAL. 1 + [6], recreation park, IX.2009, ANS.

Haplodrassus signifier (C.L. Koch, 1839)

Udmurtia [Zubko \& Roshchinenko, 1981]: fallow land.

Haplodrassus silvestris (Blackwall, 1833)

MATERIAL. 1 \& [7]: Novyi, mixed forest, 16.VI.2009, leg. ANS.

Haplodrassus soerenseni (Strand, 1900)

MATERIAL. 1 [ [7]: Volkovskii, bog, VI.2009, leg. ANS.

Micaria pulicaria (Sundevall, 1831)

MATERIAL. 1 \% [10], multiherbaceous meadow, 10.VII.09, leg. ANS

Zelotes subterraneus (C.L. Koch, 1833)

MATERIAL. 1 [7]: Novyi, small-leaved forest, 12.VI.2010, leg. ANS; $1 \sigma^{7}$, same locality, mixed forest, 2.VII.2010, leg. ANS.

\section{LINYPHIIDAE}

Abacoproeces saltuum (L. Koch, 1872)

MATERIAL. $1 \sigma^{7}$ [7]: Novyi, Pinus forest, pitfall-traps, 12.VI.2010, leg. ANS; 1 O [7]: Nechkino, mixed forest, pitfalltraps, VIII.2009, leg. ANS.

Agyneta innotabilis (O. Pickard-Cambridge, 1863)

MATERIAL. 1 ㅇ [7]: Novyi, Pinus forest, pitfall-traps, 12.VI. 2010, leg. ANS

Agyneta subtilis (O. Pickard-Cambridge, 1863)

MATERIAL. $10^{7}$ [9], edge of mixed forest, 8.VII.2009, leg. ANS

Asthenargus paganus (Simon, 1884)

MATERIAL. 1 + [3], Pinus forest, 15.IX.2009, leg. ANS

Bathyphantes approximatus (O. Pickard-Cambridge, 1871)

MATERIAL. 1 \% [1], birch forest, VII.2008, leg. AVII.

Bathyphantes gracilis (Blackwall, 1841)

MATERIAL. $1 \mathrm{O}^{7}$ [1], birch forest, VII.2008, leg. AVII.

Bolyphantes alticeps (Sundevall, 1833)

MATERIAL. $1 \mathrm{O}^{7}$ [7]: Siva, small-leaved forest, 10.VIII.2010, leg. ANS; $1 O^{7}$ [7]: Novyi, mixed forest, litter, 21.VIII.2009, leg. ANS; 2 우 [7]: Gol'yany, bottomland meadow, 7.VIII.2010, leg. ANS

Ceratinella brevis (Wider, 1834)

Udmurtia [Zubko \& Roshchinenko, 1981]: fallow land.

Diplostyla concolor (Wider, 1834)

MATERIAL. $10^{7}, 1+$ [1], birch forest, VII.2008, leg. AVII.

Udmurtia [Zubko \& Roshchinenko, 1981]: Picea-Betula forest, fallow land, field of wheat. 
Dismodicus bifrons (Blackwall, 1841)

MATERIAL. $1 \sigma^{\top}$ [7]: Nechkino, mixed forest, pitfall-traps, 9.VI.2009, leg. ANS

Drapetisca socialis (Sundevall, 1833)

MATERIAL. 1 9 [7]: Doksha, bank of river, 11.IX.2010, leg ANS; 1 + [4], mixed forest, 06.X.2009, leg. ANS

Udmurtia [Zubko \& Roshchinenko, 1981]: Picea-Betula \& Pinus forests.

Erigone atra Blackwall, 1833

Udmurtia [Zubko \& Roshchinenko, 1981]: Picea-Betula \& Pinus forests, forest meadow, field of potatoes.

Erigone dentipalpis (Wider, 1834)

MATERIAL. 1 \% [1], kitchen garden, VII.2008, leg. AVIl.

Udmurtia [Zubko \& Roshchinenko, 1981]: fallow land, field of wheat.

Gongylidium rufipes (Linnaeus, 1758)

MATERIAL. $2 \sigma^{7} \sigma^{7}$ [1], kitchen garden, VII.2008, leg. AVIl; 1 subadult $O^{7}, 1$ subadult + [7]: Siva, small-leaved forest, 10.VIII 2010, leg. ANS

Helophora insignis (Blackwall, 1841)

MATERIAL. $1 \sigma^{7}$ [1], birch forest, VII.2008, leg. AVIl; 2 $O^{7} O^{7}, 1$ ㅇ [7]: Siva, small-leaved forest, 24.VIII.2009, 10.VIII.2010, leg. ANS; 1 \% [7]: Gol'yany, bottomland meadow, 7.VIII.2010, leg. ANS.

Hylyphantes graminicola (Sundevall, 1830)

MATERIAL. $1 \sigma^{7}$ [7]: Doksha, bank of river, 11.IX.2010, leg. ANS.

Hypomma bituberculatum (Wider, 1834)

MATERIAL. 1 9 [7]: Novyi, bottomland meadow, 21.VI.2009, leg. ANS.

Kaestneria pullata (O. Pickard-Cambridge, 1863)

MATERIAL. 1 \% [7]: Volkovskii, Pinus forest, 18.VI.2009, leg. ANS.

Linyphia hortensis Sundevall, 1830 ANS.

MATERIAL. 1 + [7]: Novyi, Pinus forest, 17.VI.2009, leg.

Linyphia triangularis (Clerck, 1758)

MATERIAL. 1 [ [3], Larix forest, canopy, 15.IX.2009, leg. ANS; 1 ㅇ [7]: Siva, fire station, 24.VIII.2009, leg. ANS; $50^{7} 0^{7}, 3$ 우, same locality, small-leaved forest, 10.VIII.2010, leg. ANS; 1 $\sigma^{7}, 6$ 오 [7]: Novyi, mixed forest \& dry meadow on bank Kama River, 19-21.VIII.2009, leg. ANS; 1 OT $^{7} 4$ 우 [7]: Gol'yany, bottomland meadow, 07.VIII.2010, leg. ANS

Udmurtia [Zubko \& Roshchinenko, 1981]: Picea-Betula \& Pinus forests, forest meadow.

Microlinyphia pusilla (Sundevall, 1830)

MATERIAL. $10^{7}$ [1], agricultural meadow, VII.2008, leg. AVII.

Microneta viaria (Blackwall, 1841)

MATERIAL. 2 우 [7]: Novyi, mixed forest, litter, 16.VI.2009, leg. ANS.

Neriene clathrata (Sundevall, 1830)

MATERIAL. 1 , [1], dry multiherbaceous meadow, VII.2008, leg. AVIl; 1 ㅇ [7]: Nechkino, mixed forest, 19.VIII.2009, leg. ANS
Neriene emphana (Walckenaer, 1802)

MATERIAL. 1 \% [7]: Novyi, Pinus forest, VII.2008, leg. AVIl; 1 subadult $O^{2}, 1$ subadult $O$, same locality, Pinus forest with Picea and Betula, 19.VI.2009, leg. ANS; 1 , same locality, earthen bank of a water basin, 19.VIII.2009, leg. ANS; $1 \bigcirc^{7}$, same locality, Pinus forest, 12.VI.2010, leg. ANS; 5 우, same locality, mixed forest, 19.VIII.2009, 2.VII.2010, leg. ANS.

Udmurtia [Zubko \& Roshchinenko, 1981]: Picea-Betula \& Pinus forests.

Neriene montana (Clerck, 1758)

MATERIAL. $2 \sigma^{7} \sigma^{7}$ [1], kitchen garden, VII.2008, leg. AVIl; 2 subadult $O^{7} O^{7}$ [3], Sphagnum bog, 15.IX.2009, leg. ANS; 2 O $^{7}$ [7]: Siva, Salix thicket, 15.VI.2008, leg. ANS; 4 우 [7]: Novyi, mixed forest, bottomland meadow \& microcave in bank of a water basin, 17-21.VI.2009, leg. ANS; 5 +o, same locality, earthen bank of a water basin, 2.VII \& 19.VIII.2009, leg. ANS; 1 subadult $0^{\text {Th, }}$ same locality, mixed forest, 19.VIII.2009, leg. ANS; 4 우우 [7]: Makarovo, bank of river, 10.VI.2009, leg. ANS;

Neriene peltata (Wider, 1834)

MATERIAL. 1 9 [5], multiherbaceous meadow, VI.2010, leg. ANS; 1 [ [7]: Nechkino, mixed forest, 08.VI.2009, leg. ANS

Neriene radiata (Walckenaer, 1842)

MATERIAL. $20^{7} \sigma^{7}$ [7]: Novyi, Sphagnum bog, 19.VI.2009, leg. ANS; 1 , same locality and biotope, 18.VIII.2009, leg. ANS; 3 우, same locality, Pinus forest with green moss, 19.VI.2010, leg. ANS

Oedothorax apicatus (Blackwall, 1850)

MATERIAL. $3 O^{7} \sigma^{7}, 1+$ [1], kitchen garden, VII.2008, leg. AVIl.

Oedothorax retusus (Westring, 1851)

MATERIAL. 1 \% [2], kitchen-garden, VIII.2009, leg. ANS.

Udmurtia [Zubko \& Roshchinenko, 1981]: Picea-Betula forest, forest meadow, field of wheat, fallow land.

\section{Panamomops mengei Simon, 1926}

MATERIAL. 1 + [7]: Novyi, mixed forest, litter, 16.VI.2009, leg. ANS

Pityohyphantes phrygianus (C.L. Koch, 1836)

MATERIAL. $1 \mathrm{O}^{\mathrm{T}}$ [1], VII.2008, leg. AVII.

Poeciloneta variegata (Blackwall, 1841) ries".

Udmurtia [Zubko \& Roshchinenko, 1981]: "artificial territo-

Porrhomma pygmaeum (Blackwall, 1834)

MATERIAL. 2 o $\sigma^{7}$ [7]: Siva, bottomland Quercus forest, 15.VI.2008, leg. ANS; $1 \sigma^{7}$ [7]: Makarovo, bank of river, 10.VI.2009, leg. ANS.

Praestigia kulczynskii Eskov, 1979

MATERIAL. 1 o [7]: Siva, bottomland Quercus forest, 15.VI.2008, leg. ANS.

Silometopus elegans (O. Pickard-Cambridge, 1872)

MATERIAL. $10^{7}$ [7]: Makarovo, bank of river, 10.VI.2009, leg. ANS

Silometopus reussi (Thorell, 1871)

MATERIAL. 1 ㅇ [2], 9.V.2010, leg. ANS.

Tenuiphantes nigriventris (C.L. Koch, 1879)

MATERIAL. 1 \% [3], Sphagnum bog, 15.IX.2009, leg. ANS. 
Tenuiphantes tenebricola (Wider, 1834)

MATERIAL. $10^{7}$ [7]: Siva, small-leaved forest, 10.VIII.2010, leg. ANS.

Trematocephalus cristatus (Wider, 1834)

MATERIAL. 1 \% [7]: Siva, bottomland meadow, 15.VI.2008, leg. ANS; $10^{7}$ [7]: Novyi, dry meadow, 10.VI.2010, leg. ANS.

Walckenaeria mitrata (Menge, 1868)

MATERIAL. $10^{7}$ [7]: Novyi, small-leaved forest, pitfall-traps, 12.VI.2010, leg. ANS

\section{LIOCRANIDAE}

Agroeca brunnea (Blackwall, 1833)

Udmurtia [Zubko \& Roshchinenko, 1981]: Pinus forest

\section{LYCOSIDAE}

Acantholycosa lignaria (Clerck, 1758)

MATERIAL. $1 \sigma^{7}$ [7]: Makarovo, bank of river, 10.VI.2009, leg. ANS.

Allohogna singoriensis (Laxman, 1770)

Kizner, Syumsi, Malaja Purga, Sarapul, Votkinsk and YakshurBod'ya Districts [Krulikovskiy, 1892, 1908: as Lycosa latreillei Zubko \& Roshchinenko, 1981: as Lycosa; Zubko, 2001: as Lycosa]

Alopecosa aculeata (Clerck, 1758)

MATERIAL. $1 \sigma^{7}$ [7]: Nechkino, Pinus forest, pitfall-traps, VIII.2009, leg. ANS

Alopecosa cuneata (Clerck, 1757)

MATERIAL. $10^{7}$ [6], recreation park, IX.2009, ANS.

Udmurtia [Zubko \& Roshchinenko, 1981]: forest meadow, fallow land, field of wheat.

\section{Alopecosa inquilina (Clerck, 1758)}

MATERIAL. 1 + [7]: Novyi, dry meadow, 20.VI.2009, leg. ANS.

Alopecosa pulverulenta (Clerck, 1758)

MATERIAL. $1 \mathrm{O}^{7}$ [1], dry multiherbaceous meadow, VII.2008, leg. AVIl.

Udmurtia [Zubko \& Roshchinenko, 1981]: Alnus forest, bank of lake, fallow land, field of wheat.

Alopecosa sulzeri (Pavesi, 1873) ANS

MATERIAL. 1 [7]: Novyi, dry meadow, 12.VI.2010, leg.

Arctosa cinerea (Fabricius, 1777)

MATERIAL. 2 우 [7]: Doksha, bank of river, 20.VI.2008, leg. ANS; 1 + , Vavozh District, Gulyaevo Village, sandy spit of Kil'mez River, 1.IX.2009, leg. S.V. Dedyukhin. bank.

Udmurtia [Zubko \& Roshchinenko, 1981]: bank of lake, sand-

Arctosa perita (Latreille, 1799)

Syumsi Distric [Zubko \& Roshchinenko, 1981; Zubko, 2001]: sand-bank

Hygrolycosa rubrofasciata (Ohlert, 1865)

MATERIAL. $1 \bigcirc^{7}$ [3], Pinus forest, 15.IX.2009, leg. ANS

Pardosa agrestis (Westring, 1861)

MATERIAL. $1 \sigma^{7}, 2$ 우 [1], kitchen garden, VII.2008, leg. AVIl; $1 O^{7}$ [5], multiherbaceous meadow, VII.2010, leg. ANS; $10^{7}$
[7]: Doksha, bank of river, 20.VI.2008, leg. ANS; $10^{7}$ [7]: Siva, bottomland meadow, 15.VI.2008, leg. ANS; $2 \sigma^{7} \sigma^{7}$ [7]: Novyi, Pinus forest, pitfall-traps, 12.VI.2010, leg. ANS.

Udmurtia [Zubko \& Roshchinenko, 1981]: fallow land, wheat $\&$ potatoes fields.

\section{Pardosa agricola (Thorell, 1856)}

MATERIAL. 1 + [7]: Novyi, kitchen-garden, 15.VI.2009, leg. ANS

Pardosa amentata (Clerck, 1758)

MATERIAL. $110^{7} \sigma^{7}, 17$ 우 [1], kitchen garden, VII.2008, leg. AVIl; 1 ㅇ, [2], 9.V.2010, leg. ANS; 1 ㅇ, [5], multiherbaceous meadow, VII.2010, leg. ANS; $1 \mathrm{O}^{-1}$ [7]: Novyi, meadow, 12.VI.2010, leg. ANS.

Udmurtia [Zubko \& Roshchinenko, 1981]: Alnus forest.

Pardosa fulvipes (Collett, 1876)

MATERIAL. $140^{7} \sigma^{7}, 16$ 오 (PSU-5310) [1], agricultural meadows, birch forest, kitchen garden, VII.2008, leg. AVIl; $10^{7}, 5$ 우우 [5], multiherbaceous meadow, VI.2010, leg. ANS; 2 우우 [7]: Siva, small-leaved forest, 24.VIII.2009, 9.VIII.2010, leg. ANS; 1 $\mathrm{O}^{7}, 3$ 우, same locality, bottomland meadow, 15.VI.2009, 10.VIII. 2010, leg. ANS; 3 우 [7]: Novyi, bottomland meadow \& bottomland Quercus forest, 21.VI.2009, leg. ANS; 1 , same locality, dry meadow, 18.VIII.2009, leg. ANS; 4 우 [9], edge of mixed forest, bank of lake, 8.VII.2009, leg. ANS

Udmurtia [Zubko \& Roshchinenko, 1981]: bank of lake, fallow land, field of potatoes.

Pardosa lugubris (Walckenaer, 1802)

MATERIAL. $1 \sigma^{7}$ [6], recreation park, IX.2009, ANS; 17 오우 [7]: Siva, small-leaved forest, 24.VIII.2009, 9-10.VIII.2010, leg. ANS; $1 \bigcirc^{7}, 10$ 우 [7]: Novyi, bottomland Quercus forest, Pinus forest, Pinus forest with Picea and Betula, 17-21.VI.2009, leg. ANS; $6 O^{7} \sigma^{7}, 2$ + 9 , same locality, Pinus forest, small-leaved forest, earthen breakage of a water basin, pitfall-traps, 10-12.VI.2010, leg. ANS; 1 + [7]: Volkovskii, Pinus forest, 18.VI.2009, leg. ANS; 1 [7]: Nechkino, bottomland meadow, 9.VI.2009, leg. ANS; 13 $\sigma^{\top} \sigma^{2}$, same locality, Pinus \& mixed forests, pitfall-traps, VIII.2009, leg. ANS

Udmurtia [Zubko \& Roshchinenko, 1981]: Picea-Betula \& Pinus forests, forest meadow.

\section{Pardosa paludicola (Clerck, 1758)}

MATERIAL. $2 \sigma^{7} \sigma^{7}, 2$ 우 [1], kitchen garden, VII.2008, leg. AVIl; 1 + , [7]: Makarovo, bank of river, 10.VI.2009, leg. ANS; 1 , [7]: Nechkino, bottomland meadow, 9.VI.2009, leg. ANS.

Udmurtia [Zubko \& Roshchinenko, 1981]: Alnus forest, bank of lake.

Pardosa palustris (Linnaeus, 1758)

MATERIAL. 1 [ [1], kitchen garden, VII.2008, leg. AVIl; $10^{7}$ [5], multiherbaceous meadow, VI.2010, leg. ANS; 2 우 [7]: Siva, small-leaved forest, 15.VI.2008, 10.VIII.2010, leg. ANS; 2 우 [7]: Novyi, bottomland meadow \& dry meadow, 10-12.VI.2010, leg. ANS; 2 우 [7]: Nechkino, mixed forest, pitfall-traps, 9.VI.2009, leg. ANS; 1 [ [9], bank of lake, 8.VII.2009, leg. ANS; $10^{7}$ [10], multiherbaceous meadow, 10.VII.09, leg. ANS.

Udmurtia [Zubko \& Roshchinenko, 1981]: Alnus forests, fallow land, wheat \& potatoes fields.

Pardosa plumipes (Thorell, 1875)

MATERIAL. $10^{7}$ [7]: Siva, bottomland Quercus forest, 15.VI.2008, leg. ANS; $1 \bigcirc^{7}, 3$ 우 [7]: Novyi, bottomland meadow, 21.VI.2009, 12.VI.2010, leg. ANS.

Udmurtia [Zubko \& Roshchinenko, 1981]: field of wheat, fallow land. 
Pardosa prativaga (L. Koch, 1870)

MATERIAL. $60^{7} 0^{7}, 7$ 우 [7]: Siva, bottomland meadow, bank of river, 15.VI.2008, 10.VIII.2010, leg. ANS; 1 , same locality, small-leaved forest, 10.VIII.2010, leg. ANS; $10^{\prime \prime}, 5$ 우 [7]: Novyi, bottomland meadow, 21.VI.2009, leg. ANS; 1 o , 2 우 [7]: Volkovskii, bottomland oak forest, coastal vegetation, 20.VI.2009, leg. ANS. of lake.

Udmurtia [Zubko \& Roshchinenko, 1981]: Alnus forest, bank

Pardosa schenkeli Lessert, 1904 ANS.

MATERIAL. 3 우 [7]: Novyi, dry meadow, 10.VI.2010, leg.

Pardosa sphagnicola (Dahl, 1908)

MATERIAL. 1 + [5], multiherbaceous meadow, VI.2010, leg. ANS; 1 + [7]: Novyi, Sphagnum bog, 19.VI.2009, leg. ANS; 10 " same locality, mixed forest, 21.VIII.2009, leg. ANS; $10^{7}, 6$ 우 [7]: Volkovskii, bog, 10.VI.2010, leg. ANS; 1 + [9], Pinus forest, 7.VII.2009, leg. ANS.

Pirata hygrophilus Thorell, 1872

MATERIAL. 1 i [1], agricultural meadows, VII.2008, leg. AVIl; 1 + [3], Sphagnum bog, 15.IX.2009, leg. ANS; 1 subadult $O^{7}$ [4], mixed forest, 6.X.2009, leg. ANS.

Pirata piraticus (Clerck, 1758)

MATERIAL. $10^{7}$ [7]: Novyi, bottomland meadow, 21.VI.2009, leg. ANS; 2 우 [7]: Volkovskii, coastal vegetation, 20.VI.2009, leg. ANS.

Pirata piscatorius (Clerck, 1758)

MATERIAL. 1 ㅇ [9], edge of mixed forest, 8.VII.2009, leg. ANS.

Udmurtia [Zubko \& Roshchinenko, 1981]: bank of lake.

Tricca lutetiana (Simon, 1876)

MATERIAL. 1 + [7]: Siva, fire station, 24.VIII.2009, leg. ANS

Trochosa ruricola (DeGeer, 1778)

MATERIAL. $7 \sigma^{7} \sigma^{7}, 5$ 우 [1], agricultural meadows, kitchen garden, VII.2008, leg. AVIl; 1 \%, [2], 9.V.2010, leg. ANS; 1 \% [7]: Siva, bottomland meadow, 15.VI.2008, leg. ANS; $1 \mathrm{O}^{7}, 1$ ㅇ, same locality, small-leaved forest, 10.VIII.2010, leg. ANS.

Udmurtia [Zubko \& Roshchinenko, 1981]: bank of lake, fallow land, wheat \& potatoes fields.

Trochosa spinipalpis (F.O. Pickard-Cambridge, 1895)

MATERIAL. 1 \% [7]: Volkovskii, coastal vegetation, 20.VI. 2009, leg. ANS.

Trochosa terricola Thorell, 1856

MATERIAL. 1 + [7]: Siva, mixed forest, 20.VI.2009, leg. ANS; 1 P, same locality, small-leaved forest, 10.VIII.2010, leg. ANS; 1 [ [7]: Volkovskii, bottomland oak forest, 20.VI.2009, leg. ANS; $2 O^{7} \sigma^{7}, 4$ 우 [7]: Nechkino, mixed forest, pitfall-traps, 10.VIII.2010, leg. ANS.

Xerolycosa miniata (C.L. Koch, 1834)

MATERIAL. $4 \sigma^{7} \sigma^{7}, 1$ o (PSU-5311), [1], kitchen garden, VII.2008, leg. AVIl; 1 O' [7]: Doksha, bank of river, 23.VI.2009, leg. ANS; $3 \bigcirc^{7} O^{7}, 1$ ㅇ [7]: Novyi, bank of river, 21.VI.2009, leg. ANS; $1 \sigma^{\prime}, 2+$, same locality, bottomland \& dry meadows, earthen breakage of a water basin, 10-12.VI.2010, leg. ANS.

Udmurtia [Zubko \& Roshchinenko, 1981]: fallow land.

Xerolycosa nemoralis (Westring, 1861)

MATERIAL. $8 \sigma^{7} \sigma^{7}, 11$ oᄋ [7]: Novyi, mixed forest, Pinus forest, meadow, 16-20.VI.2009, 12.VI \& 2.VII.2010, leg. ANS; 1 $\sigma^{7}$ [7]: Volkovskii, bog, 12.VI.2010, leg. ANS; 1 [7]: Gol'yany, dry lichen-grass association on sand, 7.VIII.2010, leg. ANS.

\section{METIDAE}

Metellina merianae (Scopoli, 1763)

MATERIAL. 1 ㅇ [7]: Novyi, earthen bank of a water basin, 19.VIII.2009, leg. ANS.

\section{Metellina segmentata (Clerck, 1758)}

MATERIAL. 1 9 [7]: Doksha, bank of river, 11.IX.2010, leg. ANS; $7 O^{7} \sigma^{7}, 1$, same locality small-leaved forest, 10.VIII.2010, leg. ANS; $1+$, same locality, bottomland meadow, 10.VIII.2010, leg. ANS; $12{\sigma^{7}}^{7}, 13$ 우 [7]: Novyi, dry meadow on bank of Kama River, earthen bank of a water basin, 19-21.VIII.2009, leg. ANS; 2 우 [7]: Gol'yany, bottomland meadow, 7.VIII.2010, leg. ANS.

Udmurtia [Zubko \& Roshchinenko, 1981]: Pinus forest, forest meadow.

\section{OXYOPIDAE}

Oxyopes ramosus (Martini \& Goeze, 1778)

MATERIAL. 1 (PSU-5313) [1], dry multiherbaceous meadows, VII.2008, leg. AVIl; 4 juv. [7]: Novyi, dry meadow \& Sphagnum bog, 18.VIII.2009, leg. ANS; 1 juv. [7]: Volkovskii, shrub on edge of bog, 18.VI.2009, leg. ANS; 1 juv. [7]: Gol'yany, dry lichen-grass association on sand, 7.VIII.2010, leg. ANS.

\section{PHILODROMIDAE}

Philodromus cespitum (Walckenaer, 1802)

MATERIAL. $10^{7}$ [1], dry multiherbaceous meadow, VII.2008, leg. AVIl; $20^{7} 0^{7}, 2$ 우 [7]: Novyi, bottomland meadow, bottomland Quercus forest, kitchen garden, 16-21.VI.2009, leg. ANS; 1 $\sigma^{7}$, same locality, dry meadow, 12.VI.2010, leg. ANS; $1 \sigma^{7}, 1$ ㅇ [7]: Volkovskii, Pinus \& Quercus forests, 18-20.VI.2009, leg. ANS; 1 $\sigma^{\curvearrowright}$, [7]: Nechkino, mixed forest, pitfall-traps, 9.VI.2009, leg. ANS.

Philodromus emarginatus (Schrank, 1803)

MATERIAL. $1 \sigma^{7}$ [7]: Nechkino, bottomland meadow, 9.VI.2009, leg. ANS; 1 ㅇ [9], edge of mixed forest, 8.07.09, leg. ANS; 1 juv. [7]: Novyi, dry meadow, 21.VI.2009, leg. ANS.

Philodromus fuscomarginatus (DeGeer, 1778) ANS.

MATERIAL. 1 O $^{7}$ [7]: Novyi, Sphagnum bog, 18.VI.2008, leg.

Philodromus histrio (Latreille, 1819)

MATERIAL. $1 \sigma^{7}$, Kambarka Town, waste ground, 12.IX.2010, leg. S.V. Dedyukhin.

Udmurtia [Zubko \& Roshchinenko, 1981]: Pinus forest, forest meadow.

Philodromus poecilus (Thorell, 1872) ANS.

MATERIAL. 1 O [7]: Siva, bottomland meadow, VI.2007, leg.

Thanatus formicinus (Clerck, 1758)

MATERIAL. 1 \% [7]: Novyi, bottomland Quercus forest, 21.VI.2009, leg. ANS.

Thanatus sabulosus (Menge, 1875)

MATERIAL. $1 O^{7}$ [7]: Novyi, Pinus forest with green moss, 16.VI.2009, leg. ANS 
Tibellus maritimus (Menge, 1875)

MATERIAL. $1 \sigma^{7}$ [7]: Siva, bottomland meadow, VI.2007, leg. ANS; 2 우 [7]: Novyi, bottomland meadow, 21.VI.2009, leg. ANS; $3 \sigma^{7} \sigma^{7}, 3$ 우 [7]: Volkovskii, coastal vegetation, shrub on edge of bog, 18-20.VI.2009, leg. ANS; 1 ○ [8], meadow bog, 21.VI.2007, leg. ANS; 1 + [9], bog, 8.VII.2009, leg. ANS

Tibellus oblongus (Walckenaer, 1802)

MATERIAL. $1 O^{7}$ [1], dry multiherbaceous meadow, VII.2008, leg. AVIl; 2 우 [7]: Siva, bottomland meadow, 15.VI.2008, leg. ANS; $2 \sigma^{\prime} O^{7}, 5$ 우 [7]: Novyi, dry meadow, bottomland Quercus forest, 21.VI \& 18.VIII.2009, leg. ANS; $10^{7}, 2$ 우 [7]: Volkovskii, dry meadow, bog, 10-12.VI.2010, leg. ANS; 1 [10], multiherbaceous meadow, 10.VII.09, leg. ANS; 1 , same locality, vegetation on bank, 9.VII.2009, leg. ANS.

Udmurtia [Zubko \& Roshchinenko, 1981]: Pinus forest, forest meadow.

\section{PISAURIDAE}

Dolomedes fimbriatus (Clerck, 1758)

MATERIAL. 1 + [7]: Siva, bottomland of Kama River, VI.2007, leg. ANS; 1 , same locality, bottomland meadow, VIII.2010, leg. ANS; 2 우 [7]: Volkovskii, bottomland oak forest, 20.VI.2009, leg. ANS; 1 O', 1 ㅇ [7]: Nechkino, mixed forest, pool, 9.VI.2009, leg. ANS.

Udmurtia [Zubko \& Roshchinenko, 1981]: bank of lake.

Dolomedes plantarius (Clerck, 1758)

Udmurtia [Zubko \& Roshchinenko, 1981]: bank of lake.

Pisaura mirabilis (Clerck, 1758)

MATERIAL. 1 ㅇ [9], bog, 7.VII.2009, leg. ANS.

\section{SALTICIDAE}

Aelurillus v-insignitus (Clerck, 1758)

MATERIAL. $2 O^{7} \sigma^{7}$ [7]: Novyi, dry meadow, 12.VI.2010, leg. ANS.

Ballus chalybeius (Walckenaer, 1802)

MATERIAL. 1 juv. [7]: Novyi, small-leaved forest, 10.VIII. 2010, leg. ANS.

Udmurtia [Zubko \& Roshchinenko, 1981]: Picea-Betula \& Pinus forests.

Evarcha arcuata (Clerck, 1758)

MATERIAL 1 [ [1], dry multiherbaceous meadows, VII.2008, leg. AVIl; $2 O^{7} O^{7}, 1$ ㅇ [5], multiherbaceous meadow, VI.2010, leg. ANS; 1 + [7]: Siva, bottomland meadow, 15.VI.2008, leg. ANS; 9 $\sigma^{7} \sigma^{7}, 5$ 우 [7]: Novyi, bottomland Quercus forest, bottomland \& dry meadows, Sphagnum bog, kitchen garden, 15-21.VI \& 18.VIII. 2009, leg. ANS; 8 O $^{7}, 9$ \% [7]: Volkovskii, bog, coastal vegetation, dry meadow, 12-20.VI.2009, leg. ANS; 3 ○ $^{7}$, 2 우 [7]: Gol'yany, bottomland meadow, dry lichen-grass association on sand, 7.VIII.2010, leg. ANS; 1 subadult $\sigma^{7}$ [7]: Nechkino, mixed forest, 9.VI.2009, leg. ANS; $1 \sigma^{7}$ [8], multiherbaceous meadow, 21.VII.2008, leg. ANS

Evarcha falcata (Clerck, 1758)

MATERIAL. $1 \sigma^{7}$ [7]: Doksha, bank of river, 20.VI.2008, leg. ANS; $1 \sigma^{7}$, [7]: Siva, bottomland meadow, VI.2007, leg. ANS; 1 \% [7]: Novyi, Pinus forest with green moss, 16.VI.2009, leg. ANS; 1 $\mathrm{O}^{7}$, same locality, mixed forest, 19.VIII.2009, leg. ANS; 1 subadult $\sigma^{7}$ [7]: Volkovskii, bog, 18.VI.2009, leg. ANS; $1 \sigma^{7}$, [8], multiherbaceous meadow, 21.VII.2008, leg. ANS.

Udmurtia [Zubko \& Roshchinenko, 1981]: Picea-Betula \& Pinus forests, forest meadow.
Evarcha laetibunda (C.L. Koch, 1846)

Udmurtia [Zubko \& Roshchinenko, 1981]: Picea-Betula \& Pinus forests, forest meadow.

Heliophanus auratus C.L. Koch, 1835

MATERIAL. 1 + [7]: Siva, bottomland Quercus forest, 15.VI.2008, leg. ANS; 3 우 [7]: Novyi, bottomland Quercus forest \& meadow, 21.VI.2009, leg. ANS.

Udmurtia [Zubko \& Roshchinenko, 1981]: Pinus forest, forest meadow.

Heliophanus cupreus (Wlckenaer, 1802)

Udmurtia [Zubko \& Roshchinenko, 1981]: Pinus forest.

Heliophanus flavipes (Hahn, 1832)

MATERIAL. 1 \% [7]: Volkovskii, dry meadow, 12.VI.2010, leg. ANS

Phlegra fasciata (Hahn, 1826)

MATERIAL. $10^{7}$ [7]: Volkovskii, dry meadow, 12.VI.2010, leg. ANS.

Pseudeuophrus erratica (Walckenaer, 1826)

Udmurtia [Zubko \& Roshchinenko, 1981: as Euophrus]: Pinus forest, forest meadow.

Salticus cingulatus (Panzer, 1797)

MATERIAL. $1 \sigma^{7}$ [7]: Siva, bottomland meadow, 15.VI.2008, leg. ANS.

Udmurtia [Zubko \& Roshchinenko, 1981]: forest meadow.

Sibianor aurocinctus (Ohlert, 1865) ANS.

MATERIAL. 1 + [7]: Novyi, dry meadow, 10.VI.2010, leg.

Sitticus dzieduszyckii (L. Koch, 1870)

MATERIAL. $1 \sigma^{7}$ [7]: Doksha, bank of river, 20.VI.2008, leg. ANS; $1 O^{7}$, same locality and biotope, 10.VIII.2010, leg. ANS; $1 O^{7}$ [7]: Novyi, bank of river, 21.VI.2009, leg. ANS; $60^{7} \sigma^{7}$, same locality, earthen breakage of a water basin, 19.VIII.2010, leg. ANS.

Sitticus floricola (C.L. Koch, 1837)

MATERIAL. 2 +P [9], edge of mixed forest, bank of lake, 8.07.2009, leg. ANS.

Sitticus terebratus (Clerck, 1758)

MATERIAL. $1 \sigma^{7}, 1$ (PSU-5317), [1], kitchen garden, VII.2008, leg. AVIl; $20^{7} \sigma^{7}$ [7]: Nechkino, bottomland meadow, on wall of shed, 14.VIII.2009, leg. ANS.

\section{SPARASSIDAE}

\section{Micrommata roseum (Clerck, 1758)}

MATERIAL. 1 ㅇ [7]: Siva, bottomland meadow, 15.VI.2008, leg. ANS; 10 , same locality, small-leaved forest, 24.VIII.2009, leg. ANS; 2 우, 1 subadult $q$ [7]: Novyi, Quercus forest, dry \& bottomland meadows, 21.VI.2009, leg. ANS; 1 juv., same locality, Sphagnum bog, 18.VIII.2009, leg. ANS.

\section{TETRAGNATHIDAE}

Pachygnatha clercki Sundevall, 1823

MATERIAL. $10^{7}$ [1], kitchen garden, VII.2008, leg. AVII. 
Pachygnatha deegeri Sundevall, 1830

MATERIAL. $20^{7} \sigma^{7}, 4$ 우 [1], kitchen garden, VII.2008, leg. AVIl; 1 o [7]: Nechkino, bottomland meadow, 9.VI.2009, leg. ANS.

Udmurtia [Zubko \& Roshchinenko, 1981]: field of wheat, fallow land, bank of lake.

Pachygnatha listeri Sundevall, 1830

MATERIAL. 1 9 [7]: Siva, bottomland of Kama River, VI.2007, leg. ANS; 2 우, same locality, bottomland Quercus forest \& bottomland meadow, 15.VI.2008, leg. ANS; 1 \%, same locality, smallleaved forest, 24.VIII.2009, leg. ANS; $10^{7}$ [7]: Novyi, Pinus forest with green moss, 16.VI.2010, leg. ANS; 1 + [7]: Nechkino, mixed forest, 9.VI.2009, leg. ANS

Tetragnatha dearmata Thorell, 1873

MATERIAL. $3 \sigma^{7} \sigma^{7}, 2$ 우 [1], kitchen garden, VII.2008, leg. AVIl; 1 [5], multiherbaceous meadow, VI.2010, leg. ANS; 2 $\sigma^{7} \sigma^{7}$ [7]: Doksha, bank of river, 20.VI.2008, leg. ANS; 1 ㅇ [7]: Siva, bottomland meadow, 15.VI.2008, leg. ANS; 3 우 [7]: Novyi, bottomland oak forest, microcave in rock, 10-21.VI.2009, leg. ANS; $5{\sigma^{7}}^{7}$, same locality, earthen bank of a water basin, 19.VIII.2009, 2.VII.2010, leg. ANS; 1 ○ [7]: Makarovo, bank of river, 10.VI.2009, leg. ANS.

Tetragnatha extensa (Linnaeus, 1758)

MATERIAL 1 juv. [7]: Doksha, bank of river, 20.VI.2008, leg. ANS; 1 [ [5], multiherbaceous meadow, VI.2010, leg. ANS; 1 juv., [7]: Siva, bottomland Ouercus forest, 15.VI.2008, leg. ANS 2 of , same locality, bottomland meadow, 15.VI.2008, 10.VIII.2010, leg. ANS; $3 O^{7} \sigma^{7}, 4$ 우 [7]: Novyi, dry meadow on bank of Kama River, bottomland meadow, 21.VI.2009, leg. ANS; 1 subadult $0^{7}$, same locality, earthen bank of a water basin, 10.VI.2010, leg. ANS; 1 , 1 juv. [7]: Volkovskii, bottomland oak forest, edge of bog, 1820.VI.2009, leg. ANS; $1 \mathrm{O}^{7}$ [9], bank of lake, 8.VII.2009, leg. ANS

Udmurtia [Zubko \& Roshchinenko, 1981]: bank of lake, forest meadow.

Tetragnatha montana Simon, 1874

MATERIAL. 1 o [7]: Siva, bottomland meadow, VI.2007, leg. ANS; $1 \mathrm{O}^{T}$ [7]: Novyi, dry meadow on bank of Kama River, 21.VI.2009, leg. ANS; 1 ㅇ, same locality, earthen bank of a water basin, 2.VII.2010, leg. ANS.

Tetragnatha nigrita Lendl, 1886

MATERIAL. $10^{7}, 2$ 우 (PSU-5312) [1], Pinus forest, VII.2008, leg. AVIl.

Tetragnatha obtusa C.L.Koch, 1837

MATERIAL. $1 \sigma^{7}, 1$ [1], dry multiherbaceous and agricultural meadows, VII.2008, leg. AVIl; $1 \sigma^{7}$ [7]: Doksha, bank of river, 10.VI.2010, leg. ANS; 1 \% [7]: Siva, bottomland meadow, 15.VI.2010, leg. ANS; 1 O [7]: Novyi, bottomland meadow, 21.VI.2009, leg. ANS; 1 \& [7]: Volkovskii, coastal vegetation, 20.VI.2009, leg. ANS.

Tetragnatha pinicola L. Koch, 1870

MATERIAL. $30^{7} \sigma^{7}, 3$ Oᄋ [7]: Novyi, dry meadow, Pinus forest, kitchen-garden, 12-17.VI.2009, leg. ANS; 1 subadult $\sigma^{7}$, same locality, mixed forest, 19.VIII.2009, leg. ANS; 1 + 2 subadult 우 [7]: Volkovskii, shrub on edge of bog, 18.VI.2009, leg. ANS.

Udmurtia [Zubko \& Roshchinenko, 1981]: Picea-Betula \& Pinus forests, forest meadow.

\section{THERIDIIDAE}

Crustulina guttata (Wider, 1834)

MATERIAL. 1 ㅇ [7]: Novyi, mixed forest, litter, 16.VI.2009, leg. ANS.
Cryptachea riparia (Blackwall, 1834)

MATERIAL. 1 q (PSU-5314) [1], kitchen garden, VII.2008, leg. AVIl; 1 + [7]: Novyi, mixed forest, canopy, 16.VI.2009, leg. ANS

Enoplognatha ovata (Clerck, 1758)

MATERIAL. 1 [ [2], kitchen-garden, VIII.2009, leg. ANS; 1 + [9], bog, 7.VII.2009, leg. ANS; 2 +क [10], vegetation on bank, 9.VII.09, leg. ANS.

Udmurtia [Zubko \& Roshchinenko, 1981]: Picea-Betula \& Alnus forests, forest meadow.

Episinus truncatus (Latreille, 1809) AVIl

MATERIAL. 2 우 (PSU-5316) [1], birch forest, VII.2008, leg.

Euryopis flavomaculata (C.L. Koch, 1836)

MATERIAL. $10^{7}$ [7]: Novyi, small-leaved forest, pitfall-traps, 12.VI.2010, leg. ANS.

Lasaeola tristis (Hahn, 1833)

MATERIAL. 1 o [7]: Volkovskii, shrub on edge of bog, 18.V.2009, leg. ANS.

Neottiura bimaculata (Linnaeus, 1767)

MATERIAL. $1 \sigma^{7}, 1$ + [7]: Novyi, dry meadow, bottomland oak forest, 19-21.VI.2009, leg. ANS.

Parastetoda lunata (Clerck, 1758)

Udmurtia [Zubko \& Roshchinenko, 1981: as Achaearanea]: Picea-Betula \& Pinus forests.

Parasteatoda simulans (Thorell, 1875)

MATERIAL. 1 + 1 juv. [7]: Volkovskii, bottomland oak forest, 20.VI.2009, leg. ANS.

Parasteatoda tabulata (Levi, 1980)

MATERIAL. $1 \sigma^{7}, 2$ 우 [7]: Novyi, earthen bank of a water basin, 19.VIII.2009, 2.VII.2010, leg. ANS.

Phylloneta impressa (L.Koch, 1881)

MATERIAL. $1 \mathrm{O}^{7}$ [1], dry multiherbaceous meadows, VII.2008, leg. AVIl; $1 \sigma^{7}$ [7]: Doksha, bank of river, 20.VI.2008, leg. ANS;4 $\sigma^{7} \sigma^{\lambda}, 1$ [ [7]: Novyi, dry meadow, 18.VI.2009, 11.VI.2010, leg. ANS.

Phylloneta sisyphia (Clerck, 1758)

MATERIAL. 1 [7]: Novyi, Pinus forest with green moss, 16.VI.2010, leg. ANS; $10^{7}, 1$ [7]: Volkovskii, edge of bog, 18.VI.2009, leg. ANS.

Robertus lividus (Blackwall, 1836)

MATERIAL. $10^{7}[1]$, kitchen garden, VII.2008, leg. AVIl; 1 ㅇ [4], mixed forest, 6.X.2009, leg. ANS.

Steatoda albomaculata (De Geer, 1778)

MATERIAL. $1 \sigma^{7}$ [7]: Novyi, dry meadow, 10.VI.2009, leg. ANS; 1 juv. [7]: Volkovskii, bottomland oak forest, VI.2009, leg. ANS.

Steatoda bipunctata (Linnaeus, 1758)

MATERIAL. $2 \sigma^{7} \sigma^{7}$ [1], kitchen garden, VII.2008, leg. AVIl; 2 우 [2], 9.V.2010, leg. ANS; $1 O^{7}$ [4], mixed forest, 6.X.2009, leg. ANS; $1 \Im^{7}, 1$ subadult $\bigcirc^{7}, 1$ subadult 9 [7]: Novyi, earthen bank of a water basin, 02.VII.2010, leg. ANS.

Steatoda castanea (Clerck, 1758)

MATERIAL. 1 [ [1], kitchen garden, VII.2008, leg. AVIl; 19 [5], multiherbaceous meadow, VI.2010, leg. ANS; 1 ㅇ [6], IX.2009, leg. ANS; $2 O^{7} O^{\top}$ [7]: Novyi, kitchen-garden, 17.VI.2009, leg. ANS. 
Steatoda grossa (C.L. Koch, 1838)

Udmurtia [Zubko \& Roshchinenko, 1981]: building.

Theridion pictum (Walckenaer, 1802)

MATERIAL. 1 + [7]: Volkovskii, shrub on edge of bog, 18.VI. 2009, leg. ANS.

Udmurtia [Zubko \& Roshchinenko, 1981]: Pinus, Picea-Betula $\&$ Alnus forests.

Theridion varians Hahn, 1833

MATERIAL. 1 o [1], Pinus forest, VII.2008, leg. AVIl; 1 subadult $O^{7}$ [3], Larix forest, canopy, 15.IX.2009, leg. ANS; 1 , [7]: Novyi, bog, 19.VI.2009, leg. ANS; 2 ㅇ, same locality, earthen bank of a water basin, 2.VII.2010, leg. ANS

Udmurtia [Zubko \& Roshchinenko, 1981]: Picea-Betula forest, forest meadow.

\section{THOMISIDAE}

Coriarachne depressa (C.L. Koch, 1837)

Udmurtia [Zubko \& Roshchinenko, 1981]: forest meadow.

Ebrechtella tricuspidata (Fabricius, 1775)

MATERIAL. $50^{7} \sigma^{7}, 1$ \& [7]: Siva, bottomland meadow \& small-leaved forest, 10.VIII.2010, leg. ANS; $1 \sigma^{7}$ [7]: Novyi, bottomland meadow, 21.VI.2009, leg. ANS; 1 subadult $\sigma^{7}, 1$ subadult + , same locality, dry meadow, earthen breakage of a water basin, 18-19.VIII.2009, leg. ANS; 1 q [7]: Volkovskii, bottomland oak forest, 20.VI.2009, leg. ANS; $1 \mathrm{O}^{\top}$ [7]: Gol'yany, bottomland meadow, 7.VIII.2010, leg. ANS.

\section{Misumena vatia (Clerck, 1758)}

MATERIAL. $2 O^{7} \sigma^{7}, 3$ 우 [1], dry multiherbaceous meadow, kitchen garden, VII.2008, leg. AVIl; 1 O', 1 + [7]: Siva, bottomland meadow, 15.VI.2008, 10.VIII.2010, leg. ANS; 1 क [7]: Novyi, Pinus forest with Picea and Betula, 19.VI.2009, leg. ANS; 1 + , same locality, dry meadow, 21.VIII.2009, leg. ANS; 1 $\sigma^{7}$ [7]: Volkovskii, bog, 12.VI.2010, leg. ANS; 1 ㅇ [7]: Nechkino, mixed forest, 9.VI.2009, leg. ANS; 1 [8], meadow bog, 21.VII.2008, leg. ANS; 1 ㅇ [9], edge of mixed forest, 8.07.2009, leg. ANS; 1 subadult + [10], multiherbaceous meadow, 10.VII 2009, leg. ANS

Udmurtia [Zubko \& Roshchinenko, 1981]: Picea-Betula \& Pinus forests, forest meadow.

Ozyptila praticola (C.L. Koch, 1837)

MATERIAL. $1 \sigma^{7}$ [7]: Novyi, small-leaved forest, litter, 12.VI.2010, leg. ANS; 1 [ [7]: Nechkino, Pinus forest, pitfalltraps, 14.VIII.2009, leg. ANS.

Tmarus piger (Walckenaer, 1802)

MATERIAL. 1 subadult + , 2 juv. [3], Pinus \& Larix forests, canopy, 15.IX.2009, leg. ANS; 1 juv. [7]: Novyi, mixed forest, 19.VI.2009, leg. ANS; 1 subadult + , same locality, dry meadow, 18.VIII.2009, leg. ANS; 1 juv. [7]: Gol'yany, dry lichen-grass association on sand, 7.VIII.2010, leg. ANS.

Xysticus bifasciatus C.L. Koch, 1837

MATERIAL. 1 o [7]: Nechkino, bottomland meadow, 10.VI.2009, leg. ANS

Udmurtia [Zubko \& Roshchinenko, 1981]: fallow land.

Xysticus cristatus (Clerck, 1758)

MATERIAL. 1 [1], agricultural meadow, VII.2008, leg. AVIl; $1 O^{7}$ [7]: Novyi, Pinus forest with Picea and Betula, 19.VI.2009, leg. ANS; 1 + [7]: Volkovskii, of bog, 18.VI.2009, leg. ANS; 10 [7]: Nechkino, forest on edge of bog, canopy, 9.VI.2009, leg. ANS
Udmurtia [Zubko \& Roshchinenko, 1981]: Picea-Betula forest, forest meadow, fallow land.

Xysticus kochi Thorell, 1872

Udmurtia [Zubko \& Roshchinenko, 1981]: fallow land.

Xysticus lanio C.L. Koch, 1835

MATERIAL. $1 \bigcirc^{71}$ [7]: Novyi, Pinus forest with green moss, 16.VI.2009, leg. ANS.

Xysticus luctator L. Koch, 1870

MATERIAL. 1 \% [7]: Siva, small-leaved forest, 24.VIII.2009, leg. ANS; $1 \uparrow$ [7]: Novyi, edge of forest, 16.VI.2009, leg. ANS.

Xysticus sabulosus (Hahn, 1832)

MATERIAL. 1 + [7]: Gol'yany, dry lichen-grass association on sand, 7.VIII.2010, leg. ANS.

Xysticus striatipes L. Koch, 1870

MATERIAL. 1 ㅇ [7]: Novyi, dry meadow, 19.VI.2009, leg. ANS; MATERIAL. $20^{7} \sigma^{7}$, same locality and biotope, 21.VIII.2009, leg. ANS; 1 [ [7]: Volkovskii, dry meadow, 18.VI.2009, leg. ANS; 3 O $^{7} O^{7}$ [7]: Gol'yany, bottomland meadow, dry lichen-grass association on sand, 7.VIII.2010, leg. ANS.

Xysticus ulmi (Hahn, 1831)

MATERIAL. $10^{\top}, 2$ 우 [5], multiherbaceous meadow, VI.2010, leg. ANS; 1 [ [6], recreation park, IX.2009, ANS; $3 \sigma^{7} \sigma^{7}, 1$, [7]: Siva, bottomland meadow, 15.VI.2008, leg. ANS; 1 ○7, 5 우우 [7]: Novyi, bottomland \& dry meadows, mixed forest, 18-21.VI.2009, leg. ANS \& S.V. Dedyukhin; 1 [ [7]: Volkovskii, bog, 12.VI.2010, leg. ANS; $2 \sigma^{7} \sigma^{7}, 1$ ㅇ [7]: Nechkino, mixed forest, bottomland meadow, 9.VI.2009, leg. ANS; 1 + [9], edge of mixed forest, 8.VII.2009, leg. ANS

Udmurtia [Zubko \& Roshchinenko, 1981]: bank of lake, forest meadow.

\section{ULOBORIDAE}

Uloborus walckenaerius Latreille, 1806

MATERIAL. $1 \sigma^{7}$ [7]: Volkovskii, shrub on edge of bog, 18.V.2009, leg. ANS.

\section{ZORIDAE}

Zora spinimana (Sundevall, 1832)

MATERIAL. 1 ○ [7]: Nechkino, Pinus forest, pitfall-traps, VIII.2009, leg. ANS.

ACKNOWLEDGEMENTS. This project was funded by the Russian Foundation for Basic Research (grants \#\# 0804-00926-a; 09-04-01365-a; 11-04-01716-a) and by a grant of the President of the Russian Federation for the support of leading scientific schools.

\section{References}

Baranova O.G. 2002. [Local flora of Udmurtia: the analysis, checklist, protection]. Izhevsk. 199 p. [in Russian]

Esyunin S.L., Efimik V.E. 1996. Catalogue of the spiders (Arachnida, Aranei) of the Urals. Moscow: KMK Scientific Press Ltd. $229 \mathrm{p}$.

Esyunin S.L., Laetin A.M., Tselishcheva L.G., Lyapunov A.N., Tiunov A.V. 2011. To spider fauna (Arachnida, Aranei) of the Kirov Area // Arthropoda Selecta. Vol. 20. No.4. P.283-318. 
Krasnobaev Yu.P. 2004. [Catalogue of spiders (Aranei) of the Middle Volga region]. Samara. 213 p. [in Russian]

Krulikovskiy L.K. 1892. [On the appearing of the Russian tarentula, Lycosa latreillei Koch in the Vyatka Government] // Trudy Russk. entomol. obschestva. Vol.26. P.7-9 [in Russian].

Krulikovskiy L.K. 1908. [A shot sketch of the fauna of the Vyatka Government] // Pamyatnaya knizhka Vyatskoi Gubernii I calendar na 1909 g. Vyatka. P.36-69 [in Russian].

Shernin A.I. 1971. [Order Aranei - Spiders] // Zhivotnyi mir Kirovskoy oblasti. T.1. Uchenye zapiski Kirovskogo gos. Ped. inst. No.45. Kirov. P.215-221 [in Russian].
Zubko T.L. 2001. [Spider - Aranei] // Krasnaya Kniga Udmurtskoy Respubliki. Izhevsk. P.7-12 [in Russian].

Zubko T.L., Roshchinenko V.I. 1981. [To the spider fauna of some regions of Udmurtian ASSR] // Fauna i ekologiya zhivotnykh Udmurtskoy ASSR i prilezhashchikh raionov. Izhevsk: Udmurtskiy Univ. Press. P.48-57 [in Russian].

Responsible editor D.V. Logunov 